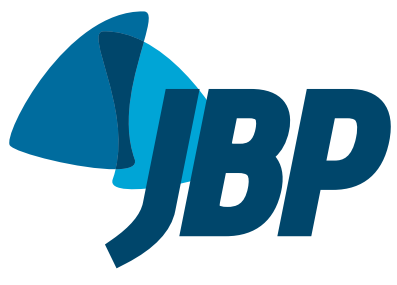

\title{
Acute invasive pulmonary aspergillosis, shortly after occupational exposure to polluted muddy water, in a previously healthy subject
}

\author{
Vikas Pilaniya', Kamal Gera', Rajesh Gothi², Ashok Shah
}

1. Department of Respiratory Medicine, Vallabhbhai Patel Chest Institute, University of Delhi, Delhi, India.

2. Department of Radiology and Imaging, Saket City Hospital, Saket, New Delhi, India.

Submitted: 23 February 2015. Accepted: 28 July 2015.

Study carried out in the Department of Respiratory Medicine, Vallabhbhai Patel Chest Institute, University of Delhi, Delhi, India.

\begin{abstract}
Invasive pulmonary aspergillosis (IPA) predominantly occurs in severely neutropenic immunocompromised subjects. The occurrence of acute IPA after brief but massive exposure to Aspergillus conidia in previously healthy subjects has been documented, although only six such cases have been reported. The diagnosis was delayed in all six of the affected patients, five of whom died. We report the case of a 50-year-old HIVnegative male, a water pipeline maintenance worker, who presented with acute-onset dyspnea and fever one day after working for $2 \mathrm{~h}$ in a deep pit containing polluted, muddy water. Over a one-month period, his general condition deteriorated markedly, despite antibiotic therapy. Imaging showed bilateral diffuse nodules with cavitation, some of which were surrounded by ground-glass opacity suggestive of a halo sign (a hallmark of IPA). Cultures (of sputum/bronchial aspirate samples) and serology were positive for Aspergillus fumigatus. After being started on itraconazole, the patient improved. We conclude that massive exposure to Aspergillus conidia can lead to acute IPA in immunocompetent subjects.
\end{abstract}

Keywords: Environmental exposure; Azoles; Water pollution; Immunocompetence; Invasive pulmonary aspergillosis.

\section{INTRODUCTION}

Fungi of the genus Aspergillus can cause a wide variety of respiratory disorders, depending on the immune status of the patient. Such disorders can range from simple allergic reactions and saprophytic colonization to invasive destruction of lung tissue with systemic dissemination, designated invasive pulmonary aspergillosis (IPA), which can be fatal. Typically, IPA occurs in immunocompromised patients, with or without neutropenia. It is now also being seen in critically ill patients, without apparent immunosuppression, in intensive care units. Other predisposing risk factors include chemotherapy (in patients with acute hematological malignancy), alcoholism, diabetes mellitus, immunosuppression therapy (in recipients of organ transplants), and high doses of systemic corticosteroids. ${ }^{(1,2)}$

There have been few reports of acute IPA caused by brief but massive exposure to Aspergillus sp. A search of the literature revealed four reports of six cases occurring in immunocompetent subjects. ${ }^{(3-6)}$ There have also been a few reports of acute IPA in immunocompetent subjects after near-drowning in contaminated water. ${ }^{(7)}$ The rarity of such scenarios prompted us to report the case of a previously healthy 50 -year-old male who developed acute IPA after working for $2 \mathrm{~h}$ in a pit containing polluted muddy water.

\section{CASE REPORT}

A 50-year-old, HIV-negative male former smoker who was a water pipeline maintenance worker with the water supply organization of the state of Delhi, India, was referred to our institute for evaluation of dyspnea and fever that had developed acutely and had peaked over a period of three days. He presented to us one month later, and his clinical course was characterized by progressive exertional dyspnea, together with cough and scant mucoid sputum, accompanied by low grade, intermittent fever with chills and rigors. There was no wheezing, chest pain, palpitations, or hemoptysis.

When questioned, the patient reported having been exposed to polluted muddy water for $2 \mathrm{~h}$ while working in a deep pit, to repair a water pipeline, one day prior to the onset of the symptoms. However, the patient emphatically denied having aspirated any of the water. The patient was completely healthy prior to this event and had no history of using corticosteroids or any other medication. He had a 10 pack-year smoking history: before quitting smoking 10 years prior, he had smoked 10 cigarettes per day for 20 years. The type of cigarette he smoked is known as a bidi (or beedi), which is manufactured in India and consists of finely ground, sun-dried tobacco rolled in a brown leaf of one of two broadleaf plants native to India-Diospyros melanoxylon or Diospyros ebenum. ${ }^{(8)}$ 
Physical examination revealed that the patient was a middle-aged man in respiratory distress. There was no pallor, digital clubbing, or cyanosis. He was tachypneic, with a respiratory rate of 25 breaths/min, and febrile. Diaphragmatic excursion, though rapid, was comparable on both sides. Breath sounds of equal intensity were audible bilaterally, together with coarse bibasilar crackles.

While breathing room air, the patient had an $\mathrm{SpO}_{2}$ of $90 \%$, with a $\mathrm{pH}$ of $7.37, \mathrm{PaCO}_{2}$ of $41.2 \mathrm{mmHg}$, and a $\mathrm{PaO}_{2}$ of $46 \mathrm{mmHg}$. The total leukocyte count was 28.6 $\times 10^{3}$ cells $/ \mu \mathrm{L}$, neutrophils accounting for $86 \%$. The results of an electrocardiogram, urinalysis, and blood glucose testing, as well as of tests of renal and hepatic function, were all within normal limits. A chest X-ray obtained after the onset of symptoms showed bilateral multifocal consolidations with cavitation in some of the lesions (Figure 1A). A contrast-enhanced HRCT scan of the chest obtained one week after the onset of symptoms revealed multiple areas of consolidation as well as nodular lesions of varying sizes with ill-defined margins. Some of those lesions showed cavitation, whereas others were surrounded by ground-glass opacity, suggestive of a halo sign (Figure 1B). An abdominal ultrasound revealed hepatomegaly with grade II fatty infiltration. Sputum smear microscopy and cultures revealed no growth of Mycobacterium tuberculosis or other aerobic organisms. However, sputum cultures showed pure growth of Aspergillus fumigatus.

Spirometry showed that the patient had an FVC of $3.01 \mathrm{~L}\left(82 \%\right.$ of predicted), an $\mathrm{FEV}_{1}$ of $1.88 \mathrm{~L}(62 \%$ of predicted), and an $\mathrm{FEV}_{1} / \mathrm{FVC}$ ratio of 0.62 . These results are indicative of moderate obstructive lung disease with no bronchodilator response. Fiberoptic bronchoscopy revealed hyperemic mucosa with whitish patches and foci of mucosal bleeding in the right upper lobe. Cultures of post-bronchoscopy sputum and bronchial aspirate showed heavy growth of $A$. fumigatus. On skin prick testing, the patient also showed immediate reactivity to $A$. fumigatus and $A$. niger. Total serum IgE was 114 kilo units of allergen per liter (kUA/L; reference: < $64 \mathrm{kUA} / \mathrm{L}$ ), and a fluorescence enzyme immunoassay (ImmunoCAP 100E; Phadia, Uppsala, Sweden) identified specific IgE and IgG antibodies against $A$. fumigatus. Multiple endobronchial and transbronchial biopsies from both lungs demonstrated chronic nonspecific inflammation. The bronchial aspirate did not yield $M$. tuberculosis or any other aerobic organism.

The patient was diagnosed with acute IPA. The diagnosis was substantiated by the heavy growth of A. fumigatus seen in the post-bronchoscopy sputum cultures, as well as in those of the bronchial aspirate. The patient was started on itraconazole $(200 \mathrm{mg}$ twice daily for two months), together with treatment for obstructive lung disease in the form of an inhaled long-acting $\beta_{2}$ agonist and an inhaled muscarinic antagonist. The patient showed remarkable clinical improvement, and chest imaging showed marked resolution of the lesions, with residual fibrosis (Figures $2 \mathrm{~A}$ and $2 \mathrm{~B}$ ). At this writing, the patient is on regular therapy for obstructive lung disease, the severity of which has been reduced considerably, and is largely asymptomatic.

\section{DISCUSSION}

Seen predominantly in immunocompromised subjects, IPA is a life-threatening form of pneumonia caused by Aspergillus spp., most commonly $A$. fumigatus. It is increasingly being seen in critically ill patients admitted to intensive care units and in recipients of organ transplants. ${ }^{(1,2)}$

The occurrence of acute IPA in a previously healthy subject is rather uncommon, and IPA due to massive but brief environmental exposure to Aspergillus sp. is quite rare; in the literature, we identified four reports describing a collective total of six cases. ${ }^{(3-6)}$
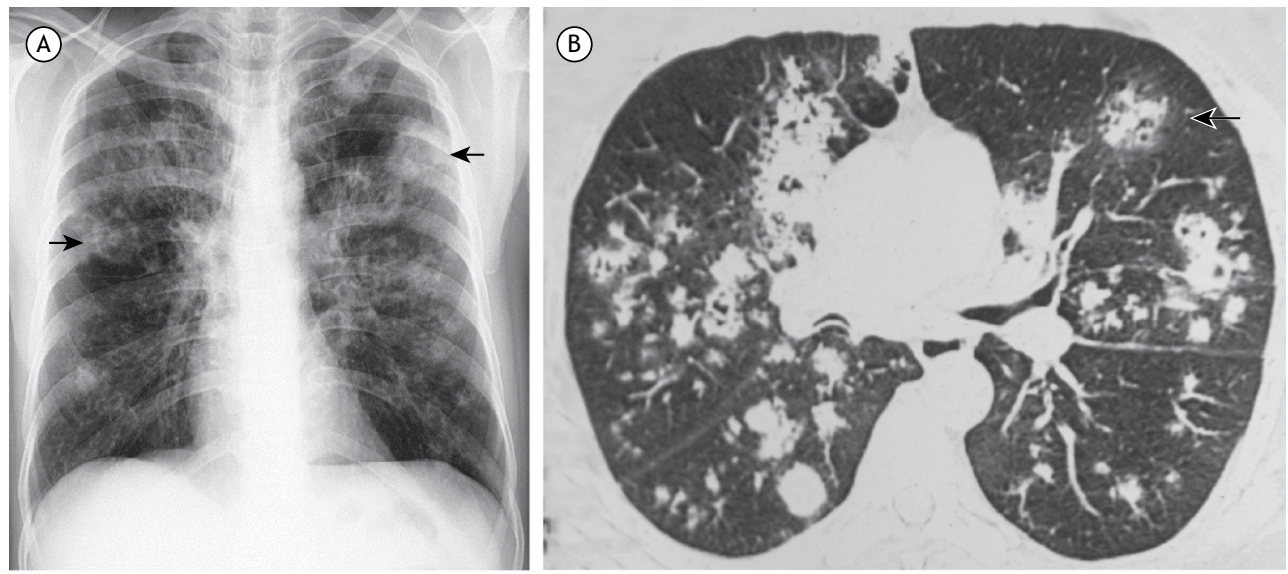

Figure 1. A) Chest X-ray, obtained after the onset of symptoms, showing bilateral multifocal consolidations with cavitation in some of the lesions (arrows); B) HRCT scan of the chest, obtained one week after the onset of symptoms, showed multiple areas of consolidation as well as nodular lesions of varying sizes, some showing cavitation and others surrounded by ground-glass opacity consistent with a halo sign (arrow). 

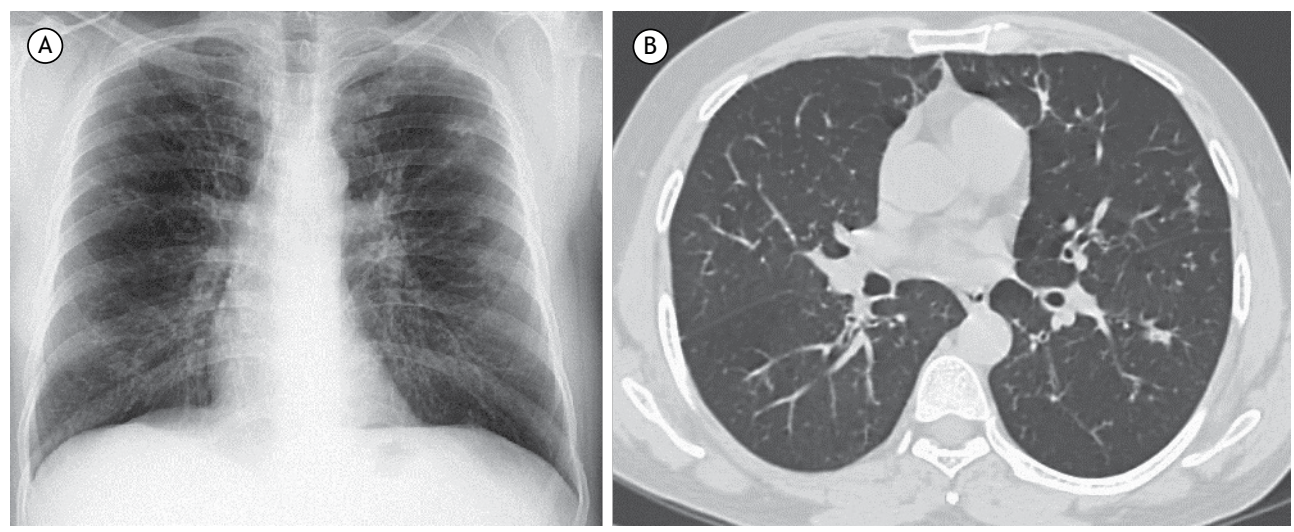

Figure 2. Chest X-ray (A) and HRCT scan of the chest (B), both obtained after two months of treatment, showing marked resolution of the lesions, with residual fibrosis.

The clinical descriptions of those six patients and of our patient are summarized in Table 1..$^{(3-6)}$ All six patients had a history of exposure to damp material likely to harbor Aspergillus sp., potentially resulting in massive inhalation of conidia. There was a considerable diagnostic delay in all six cases. Of the six patients, five died,,$^{(3-6)}$ and the diagnosis was established only at autopsy in four. ${ }^{(3,5,6)}$ Cultures of sputum or bronchial aspirate were positive for $A$. fumigatus in three of the six patients. ${ }^{(4-6)}$ In our patient, $A$. fumigatus was also cultured from sputum collected at admission. Chest $X$-rays showed patchy/homogeneous infiltrate in five patients, ${ }^{(3-6)}$ with right upper lobe cavitation in one. ${ }^{(6)}$ Our patient also showed bilateral diffuse cavitary nodules with ill-defined margins. An HRCT scan of the chest revealed bilateral interstitial infiltrates in only one of the six patients, ${ }^{(6)}$ none of the other five patients having undergone HRCT. In our patient, HRCT demonstrated multiple nodules of varying sizes with ill-defined margins, some of which showed cavitation, whereas others were surrounded by ground-glass opacity suggestive of a halo sign, which is a hallmark of IPA and therefore facilitated the diagnosis. Four of the six patients underwent biopsy ${ }^{(3,4,6)}$ : needle biopsy in one; open lung biopsy in one; and bronchial biopsy in two. Aspergillus hyphae were identified in only two of the six patients ${ }^{(4,6)}$ : from an open lung biopsy sample in one and from a bronchial biopsy sample in the other.

Fungi are known to occur in polluted water. A study conducted in Malaysia identified fungi in wastewater from a sewage treatment plant, ${ }^{\left({ }^{9}\right)}$ Aspergillus spp. being the second most common isolates. Another study, conducted in Denmark, highlighted the dangers of the inhalation of aerosols, including the conidia of Aspergillus spp., by wastewater workers. ${ }^{(10)}$ Putrid sludge from wastewater provides warm, humid conditions, which are ideal for the growth of molds. ${ }^{(10)}$ Our patient was exposed to polluted muddy water for $2 \mathrm{~h}$ while working in a deep pit. Given that his symptoms commenced the very next day, that water was most probably the source of his exposure to Aspergillus conidia.
Near-drowning episodes, especially in polluted water, can also result in acute IPA in immunocompetent individuals. ${ }^{(7)}$ Aspergillus spp. are often recovered from water bodies and are a potential cause of invasive disease in near-drowning victims, because the lung tissue can be damaged due to immersion and the large inoculum of Aspergillus that can be deposited therein under such circumstances. ${ }^{(7)}$

Our patient showed immediate skin reactivity to Aspergillus antigens, as well as testing positive for specific IgE and IgG antibodies against $A$. fumigatus, which suggests prior sensitization. Because his profession entailed working in such environments on a regular basis, it is likely that he had previously been exposed to Aspergillus antigens. Serological investigation plays a supporting role in the diagnosis of IPA. Although the disease typically occurs in immunocompromised subjects, IgG antibodies against Aspergillus antigens are detected in $29-100 \%$ of IPA patients, such sensitization being proportionally highest in non-neutropenic patients. However, the fact that it takes a mean of 10.8 days for antibodies to appear during the acute phase of the disease reduces their diagnostic utility. ${ }^{(11)}$

A halo sign is defined as a nodule or mass surrounded by ground-glass opacity on HRCT and is the earliest manifestation of IPA. This sign is transient, mainly seen during the early stages, and tends to disappear with time. ${ }^{(12)}$ It is thus imperative to obtain HRCT scans in the early stages of IPA, because this key radiological marker might not be visible thereafter. In our patient, the halo sign raised the suspicion of IPA and enabled rapid introduction of an antifungal agent, which likely helped our patient survive. Although most commonly seen in IPA, halo signs can also be seen in other fungal, viral, or bacterial infections and even in some systemic or neoplastic diseases. ${ }^{(12)}$

Because IPA usually occurs in immunocompromised hosts, it rarely comes to mind in the diagnosis of those who are immunocompetent. This frequently leads to considerable diagnostic delays and consequently to poor outcomes. Our report highlights the importance 


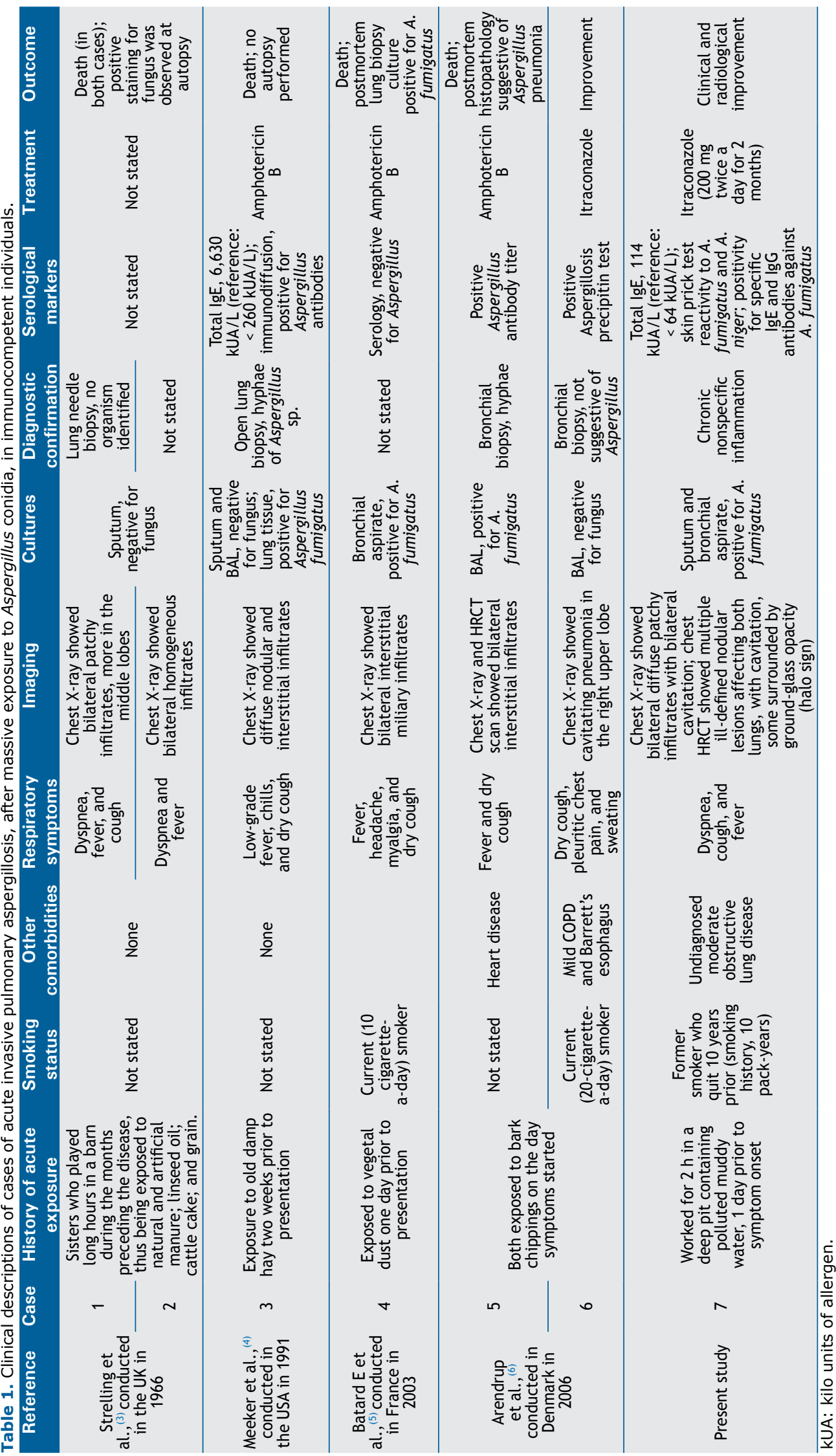


of suspecting IPA in immunocompetent patients who, in comparable scenarios, might have suffered brief but massive exposure to Aspergillus conidia, leading to acute IPA.

\section{REFERENCES}

1. Walsh TJ, Anaissie EJ, Denning DW, Herbrecht R, Kontoyiannis DP Marr KA, et al. Treatment of aspergillosis: clinical practice guidelines of the Infectious Diseases Society of America. Clin Infect Dis. 2008;46(3):327-60. http://dx.doi.org/10.1086/525258

2. Kosmidis C, Denning DW. The clinical spectrum of pulmonary aspergillosis. Thorax. 2015;70(3):270-7. http://dx.doi.org/10.1136/ thoraxjnl-2014-206291

3. Strelling MK, Rhaney K, Simmons DA, Thomson J. Fatal acute pulmonary aspergillosis in two children of one family. Arch Dis Child. 1966;41(215):34-43. http://dx.doi.org/10.1136/adc.41.215.34

4. Meeker DP, Gephardt GN, Cordasco EM Jr, Wiedemann HP. Hypersensitivity pneumonitis versus invasive pulmonary aspergillosis: two cases with unusual pathologic findings and review of the literature. Am Rev Respir Dis. 1991;143(2):431-6. http://dx.doi. org/10.1164/ajrccm/143.2.431

5. Batard E, Renaudin K, Morin O, Desjars P, Germaud P. Fatal acute granulomatous pulmonary aspergillosis in a healthy subject after inhalation of vegetal dust. Eur $\mathrm{J}$ Clin Microbiol Infect Dis. 2003;22:357-9. http://dx.doi.org/10.1007/s10096-003-0939-x

6. Arendrup $\mathrm{MC}, \mathrm{O}$ 'driscoll BR, Petersen E, Denning DW. Acute pulmonary aspergillosis in immunocompetent subjects after exposure to bark chippings. Scand J Infect Dis. 2006:38(10):945-9. http://dx.doi.org/10.1080/00365540600606580
7. Ratermann KL, Ereshefsky BJ, Fleishaker EL, Thornton AC Buch KP, Martin CA. Fulminant invasive pulmonary aspergillosis after a near-drowning accident in an immunocompetent patient. Ann Pharmacother. 2014;48(9):1225-9. http://dx.doi. org/10.1177/1060028014537611

8. Malson JL, Sims K, Murty R, Pickworth WB. Comparison of the nicotine content of tobacco used in bidis and conventional cigarettes. Tob Control. 2001;10(2):181-3. http://dx.doi.org/10.1136/tc.10.2.181

9. Fakhrul-Razi A, Alam MZ, Idris A, Abd-Aziz S, Molla AH. Filamentous fungi in Indah Water Konsortium (IWK) sewage treatment plant for biological treatment of domestic wastewater sludge. J Environ Sci Health A Tox Hazard Subst Environ Eng. 2002;37(3):309-20. http:// dx.doi.org/10.1081/ESE-120002830

10. Hansen ES, Hilden J, Klausen H, Rosdahl N. Wastewater exposure and health-a comparative study of two occupational groups. Occup Environ Med. 2003;60(8):595-8. http://dx.doi.org/10.1136/ oem.60.8.595

11. Page ID, Richardson M, Denning DW. Antibody testing in aspergillosis--quo vadis? Med Mycol. 2015;53(5):417-39. http:// dx.doi.org/10.1093/mmy/myv020

12. Georgiadou SP, Sipsas NV, Marom EM, Kontoyiannis DP. The diagnostic value of halo and reversed halo signs for invasive mold infections in compromised hosts. Clin Infect Dis. 2011:52(9):114455. http://dx.doi.org/10.1093/cid/cir122 